\title{
The Integration of RFID Technology into Business Settings ${ }^{+}$
}

\author{
María Martínez Pérez *, Carlos Dafonte and Ángel Gómez \\ CITIC-Department of Computer Science, University of A Coruña, Campus de Elviña s/n, \\ 15071 A Coruña, Spain \\ * Correspondence: maria.martinez@udc.es; Tel.: +34-981-167-000 (ext. 1264) \\ + Presented at the 2nd XoveTIC Conference, A Coruña, Spain, 5-6 September 2019.
}

Published: 22 July 2019

\begin{abstract}
At present, the term Internet of Things (IoT) is a key aspect in determining the sustainability, safety, quality and efficiency of the majority of the most important business sectors in society. The capacity to track processes that are undertaken, facilitates the recording of data of the professionals and clients involved. Identification technologies such as RFID (Radio-Frequency Identification) when integrated into sectorial activities, can generate multiple benefits.
\end{abstract}

Keywords: RFID; traceability; Internet of Things (IoT)

\section{Introduction and Objectives}

Society, is nowadays, surrounded by a multitude of new technologies that can track tasks [1] that we carry out on a daily basis. That is to say register and analyze the most important parameters of whatever process (precise identification, localization in real-time, temperature monitoring, etc.) whether it be in the elaboration or transport of a pharmaceutical, identification of a patient [2], controlling attendance or a study of the routes taken by visitors at a conference or in a museum or amusement park etc.

All of these technologies have given rise to a range of new terms in use today such as (IoT: Internet of Things) which allows for the interconnection of objects allowing for tracking of any element that requires control, providing safety, security, quality and efficiency in our daily activities.

RFID is a state-of-the-art technology that permits radiofrequency communication between a tag and a fixed reader or mobile phone and is another component within the ecosystem of IoT. RFID has different standards and functional frequencies. NFC is part of this technology and provides significant advantages. The key is to assess what RFID can contribute through introducing traceability into a particular area and analyze whether RFID is the most appropriate technology to ensure efficiency and safety in the task to be undertaken. The key parameters for decision-making and design of a successful RFID system for the selected business sector are described below.

\section{Results}

The results described as follows are based on the experience of the authors in integrating RFID technology especially into the health services area [3-7].

There are multiple configurations for an RFID system, but their way of functioning is the same. RFID technology basically enables communication between a tag and a reader through Radiofrequency. The tag will then send its identification and additional data to a reader which after reception will forward the data to the application responsible for managing it (see Figure 1). 


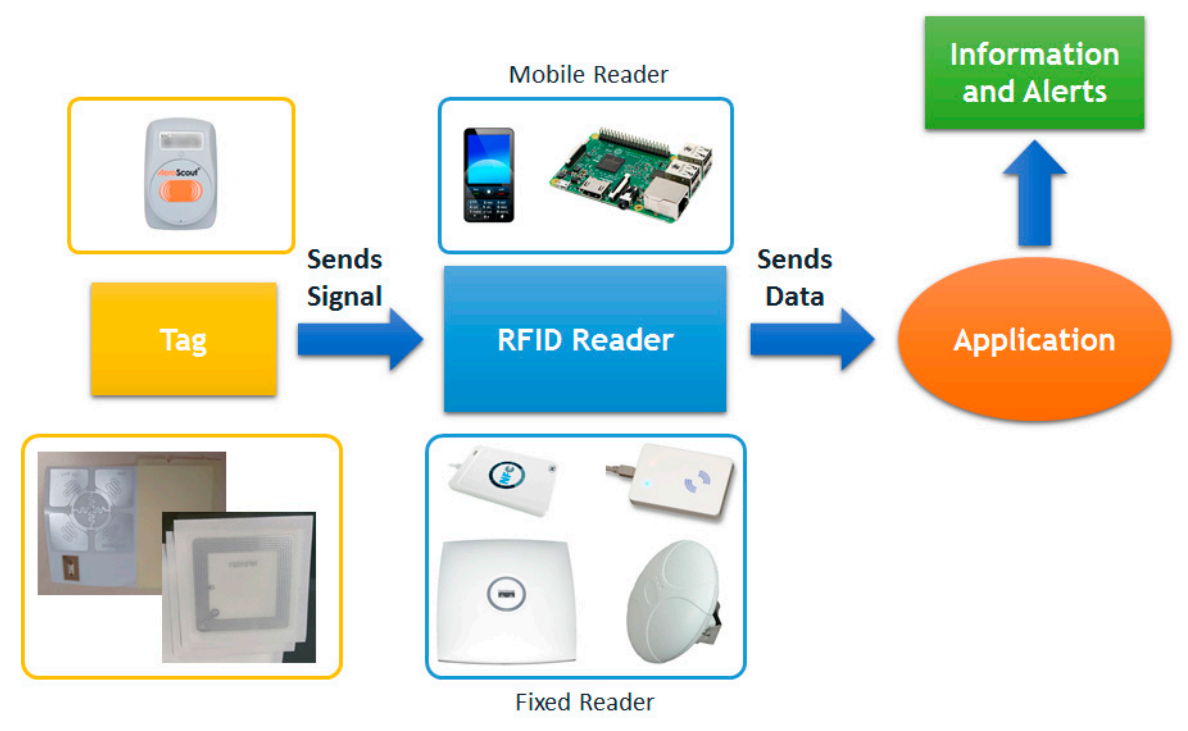

Figure 1. RFID system.

As can be seen in Figure 2, RFID is the appropriate technology to implement into a business sector if it is necessary to precisely identify and/or locate in real-time assets and/or people to be studied.

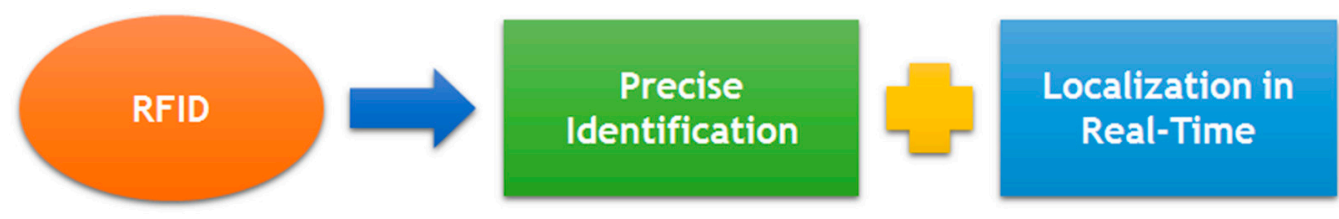

Figure 2. Principal functions of RFID technology.

The engineers responsible for implementing the RFID system have, at a minimum, to define the key parameters as shown in Figures 3 and 4 in order to obtain the technical architecture of the RFID system that is adequate for the functionalities to develop.

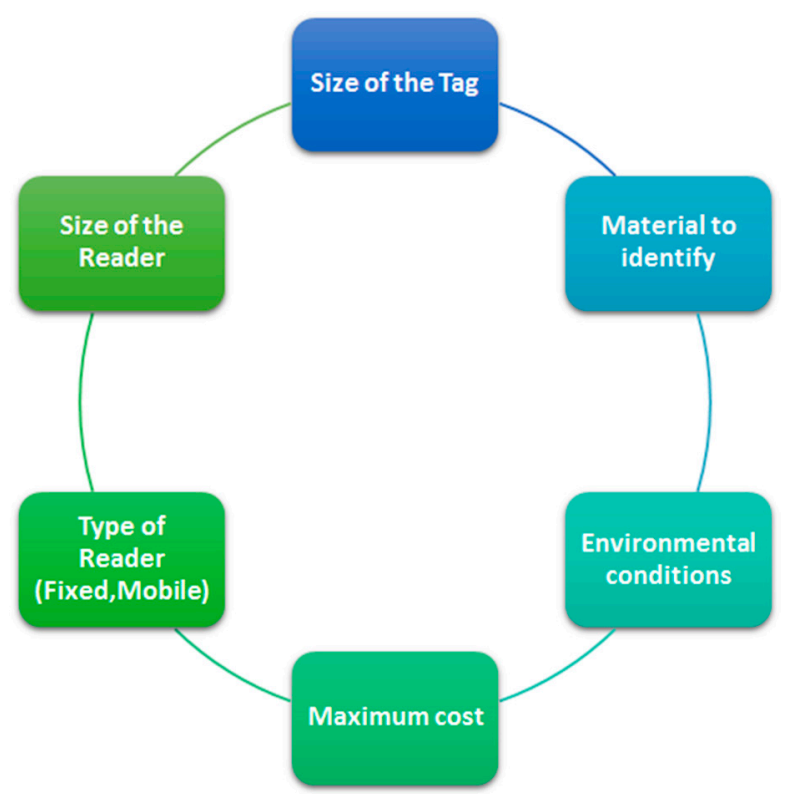

Figure 3. Key parameters in the design of an RFID system. 
The surrounding conditions and material are important to identify given that RFID technology can have interferences to its reading because of certain materials so alternatives should be considered to address these problems.

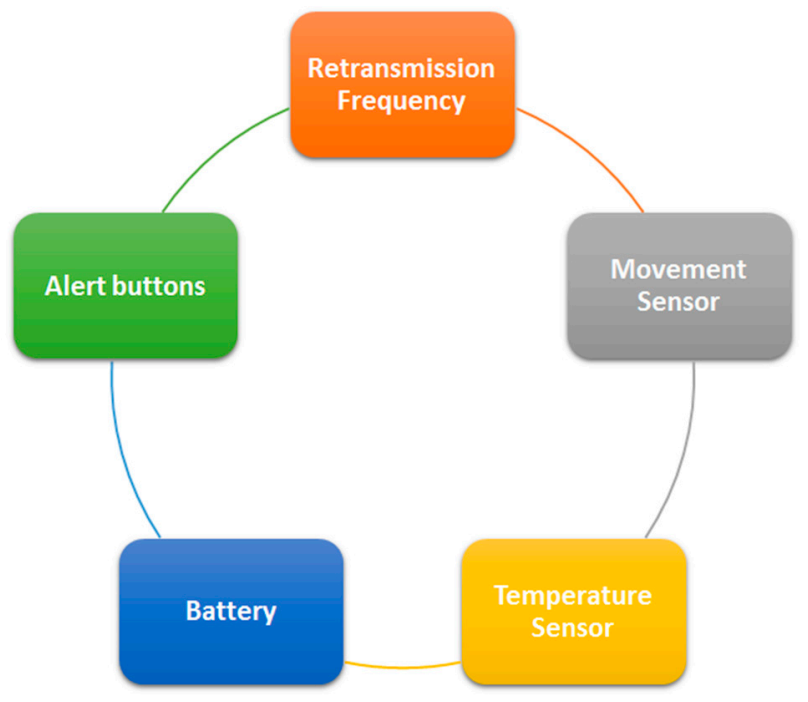

Figure 4. Key parameters in the design of an RFID system.

Most of the key parameters displayed in Figure 4 affect a key component of the RFID system: the tags. This element determines, to a large extent, the other elements so that finding the appropriate tag (in size, shape and material) assures the correct operating protocol in the process into which it is to be integrated.

To conclude, possible RFID systems in a variety of business sectors are listed below, in which the concepts previously described can be applied with the resulting improvements to the activity process:

- Tracking of products (medication, foodstuffs, organs, health products etc.); likewise the identification of transport and containers.

- Tracking of foodstuffs or gourmet products in cultivation or fishing, transport and subsequent sale and delivery (lot $\mathrm{n}^{\mathrm{o}}$, expiry date, whereabouts, times, temperature, etc.)

- Tracking of luggage in airports: identification, check-in details, avoidance of theft or recovery of items

- Tracking of animals for identification, control of protected species or domestic animals

- Tracking of documents in any area. For example medical clinical histories

- Tracking of circuits/routes in conferences, car parks, businesses, museums, hospitals, cinemas, amusement parks

- Personalized mailing of information to users about tagged objects to their mobile devices

- Management of access to congresses, car parking sites, businesses, museums, cinemas, amusement parks, etc.

- Management of payments: for example shops and tollgates

- Management of loans and returns of documents, books, and multimedia etc. For example, in libraries

- Management of stocks and expiry dates: automatic inventory in real-time in shops and warehouses

\section{Discussion and Conclusions}

The possibility of tracking business processes in a company greatly facilitates the recording of the most relevant information pertaining to it. For example, an analysis of this information may reveal potential bottlenecks so that action can be subsequently taken to avoid them or identify successful practice that, clearly, should be followed up. 
To assure the successful integration of identification technology within a sector, it is crucial to carry out a technical and economic viability study in which the results may recommend a combination of various technologies or the implementation of one IoT into those processes in which the work protocol and potential rewards justify it. RFID as a state-of- the-art technology has many capacities and applications but it must be preceded by a viability study and a design of the technical architecture of the system in which it is to be integrated.

Author Contributions: M.M.P., C.D. and Á.G. conceived, designed, and performed the experiments and developed all the tools. The paper was written by all of them.

Conflicts of Interest: The authors declare no conflict of interest

\section{References}

1. ISO 9000:2015. Sistemas de gestión de la calidad https://www.iso.org/obp/ui/es/\#iso:std:iso:9000:ed-4:v1:es (accessed on 25 June 2019)

2. Osborn, S.; Williams, S. Seven steps to patient safety. In An Overview Guide for NHS Staff, 2nd ed.; The National Patient Safety Agency: London, UK, 2004.

3. Martínez Pérez, M.; Cabrero-Canosa, M.; Hermida, J.V.; García, L.C.; Gómez, D.L.; González, G.V.; Herranz, I.M. Application of RFID technology in patient tracking and medication traceability in emergency care. J. Med. Syst. 2012, 36, 3983-3993.

4. Martínez Pérez, M.; Dafonte, C.; Gómez, Á. Traceability in Patient Healthcare through the Integration of RFID Technology in an ICU in a Hospital. Sensors 2018, 18, 1627.

5. Martínez Pérez, M.; Vázquez González, G.; Dafonte, C. Safety and Traceability in Patient Healthcare through the Integration of RFID Technology for Intravenous Mixtures in the Prescription-ValidationElaboration-Dispensation-Administration Circuit to Day Hospital Patients. Sensors 2016, 16, 1188.

6. Martínez Pérez, M.; Vázquez González, G.; Dafonte, C. Evaluation of a Tracking System for Patients and Mixed Intravenous Medication Based on RFID Technology. Sensors 2016, 16, 2031.

7. Martínez Pérez, M.; Vázquez González, G.; Dafonte, C. The Development of an RFID Solution to Facilitate the Traceability of Patient and Pharmaceutical Data. Sensors 2017, 17, 2247

(C) 2019 by the authors. Licensee MDPI, Basel, Switzerland. This article is an open access article distributed under the terms and conditions of the Creative Commons Attribution (CC BY) license (http://creativecommons.org/licenses/by/4.0/). 